\title{
Averaging model applied to the size-weight illusion
}

NORMAN H. ANDERSON 1

UNIVERSITY OF CALIFORNIA, SAN DIEGO

\begin{abstract}
A theoretical model was given for the size-weight illusion based on a principle of information integration. Judged heaviness of lifted weights was assumed to be an average of two pieces of information, weight and size, with the latter receiving negative weighting in the model formulation. Two experiments based on a Weight by Size design gave fair support to the parallelism prediction of the integration model. The hypothesis that Ss were really judging density was shown to imply a divergence prediction, contrary to the data. The theoretical analysis was generalized to include negative, positive, and comparative illusions; these were differentiated according to whether the contextual information was integrated with negative weighting, positive weighting, or served as a yardstick of judgment.
\end{abstract}

That the size of an object can influence its judged heaviness markedly is shown by the well-known size-weight illusion. Both weight and size provide relevant stimulus information, the former directly, the latter derivatively from general experience. The theoretical question considered here is how these two pieces of information are integrated to produce the judgment. An attempt will be made to answer this question with an averaging formulation that has been employed in other tasks of information integration (Anderson, 1962, 1968).

Let $s$ denote a heaviness value directly related to the actual weight, and let $s^{*}$ denote a heaviness value directly related to size. If $S$ could not see the stimulus, one would expect a judgment corresponding to s. Roughly speaking, $s^{*}$ represents an expectation based on perceived size and general experience. Let $w_{1}$ and $w_{2}$ be mathematical weights, representing the importance of the two stimulus determinants on the judgment. The model may be written,

$$
R=w_{1} s+w_{2} s^{*},
$$

where $R$ is the judged heaviness of the object as seen and lifted.

In an averaging model, the weights must sum to unity. And since a contrast effect is involved, $w_{2}$ must be negative. Equation 1 may then be written,

$$
R=(1+w) s-w s^{*} .
$$

It is also instructive to rewrite Eq. 2 in the form,

$$
R=s+w\left(s-s^{*}\right)
$$

The second term on the right then measures the illusion magnitude as a proportion of the difference between the felt weight and the expected weight. Equation 3 makes explicit that the magnitude of the illusion depends on the joint action of two factors: the size-based expectancy, $s^{*}$, and the importance, $w$, attached to it. This distinction can become important in the theoretical interpretation of various experimental manipulations.

Procedures from the theory of functional measurement (Anderson, 1970) were employed to test this formulation. The stimuli were constructed from a factorial design, while the numerical response measure allowed the application of an analysis of variance test of goodness of fit. Functional measurement can also provide interval scales of the stimulus variables as outlined below.

\section{METHOD}

Subjects made numerical judgments of heaviness of blocks that varied in size and weight. The 15 experimental blocks were constructed from a 3 by 5 , Weight by Size, factorial design. The blocks were plastic shells, approximately cubical, with side lengths of $40,48,56,65$, and $72 \mathrm{~mm}$. The interiors were weighted with lead and clay to produce the three weight values of 150 , 200 , and $250 \mathrm{~g}$. The blocks were painted black, and a hook was mounted on top for lifting.

Two end-anchors were also used: light, of $50 \mathrm{~g}$ and $35 \mathrm{~mm}$, and heavy, of $500 \mathrm{~g}$ and $88 \mathrm{~mm}$ on a side. These end-anchors were employed to minimize possible end effects in the response scale.

Two experiments were performed, both with the same design and general procedure. They differed principally in the method of lifting the weights and in the form of the response scale. The changes in the second experiment were made to eliminate a theoretical discrepancy observed in the first experiment.

\section{Experiment 1}

The $S$ was instructed to lift each block briefly, using a light, $15-\mathrm{cm}$ stick with a loop at the end to hook the weight.
Responses were ratings from 1 (lightest) to 20 (heaviest). The two end-anchors were first presented for lifting several times alternately, and $\mathbf{S}$ was told that these standards should be called 1 and 20 . The other ratings were to be used in a natural way to express how heavy each weight felt to $\mathrm{S}$ himself.

The basic stimulus replication included the 15 experimental blocks and two presentations of each end-anchor. Four replications were given, separated by short rest periods. S was told that the first replication was only practice, to give him a chance to get used to the response scale and the feel of the blocks. The stimuli were listed on 19 cards and this deck was shuffled between successive replications for each $S$ to randomize the order of presentation.

Each trial took approximately 16 to $20 \mathrm{sec}$. E placed the weight on a timer-platform, and $3 \mathrm{sec}$ later a clock signaled $S$ to lift. If the block was not returned to the platform within $2 \mathrm{sec}$, a buzzer informed $S$ that he was taking too long. Ss were 26 students who were fulfilling a class requirement in introductory psychology.

\section{Experiment 2}

This was quite similar to Experiment 1, the two major changes being in the method of lifting the weights, and giving the response. The weights were lifted by pulling down on a cylindrical handle attached to a string that led over two pulleys, separated by $25 \mathrm{~cm}$, to a counterweight and loop to catch the hook on the block. The primary purpose of this change was to get S's hand completely out of the visual field of the block.

Each response was a slash across a line on a separate paper slip. These slips were $6 \mathrm{~cm}$ high by $18 \mathrm{~cm}$ long, with a line beginning $2.5 \mathrm{~cm}$ from the left edge and extending to the right edge. This response scale was employed since associated work on psychophysical averaging had indicated that it might improve on the rating scale.

End anchors were presented as in Experiment 1. On presenting the light anchor, E told $\mathrm{S}$ to respond "about here," pointing to a region 1 to $2 \mathrm{~cm}$ from the left end of the line; similarly, the heavy anchor was to be marked 2 to $4 \mathrm{~cm}$ from the right end.

There were five presentations of the 
basic stimulus replication, separated by 2 -min rests. Trial timing gave $4 \mathrm{sec}$ for the initial visual inspection, $3 \mathrm{sec}$ for the lift. Ss were 24 students who were fulfilling a class requirement in introductory psychology.

\section{RESULTS}

Figure 1 shows a substantial size-weight illusion in both experiments. If size had no effect on the judgments, each curve would be horizontal. The observed upward slope means that a decrease in visual size caused an increase in judged heaviness.

The central question of this experiment concerns the integration of the size and weight information. The present integration model implies that the three curves in each panel of Fig. I should be parallel (Anderson, 1968, 1970). Experiment 1 shows a moderate but definite convergence to the right. The curves of Experiment 2, however, are very nearly parallel.

The statistical test of the parallelism prediction is given by the Size by Weight interaction of the analysis of variance. This was significant for Experiment 1 $[F(8,200)=2.67]$ but not for Experiment $2 \quad[F(8,184)=.83]$. These tests confirm the graphical test of parallelism.

It seems fair to say that the model shows reasonable quantitative promise. The discrepancy of Experiment 1 is significant, but not overly large. It may simply reflect methodological shortcomings that were removed by the procedural changes of

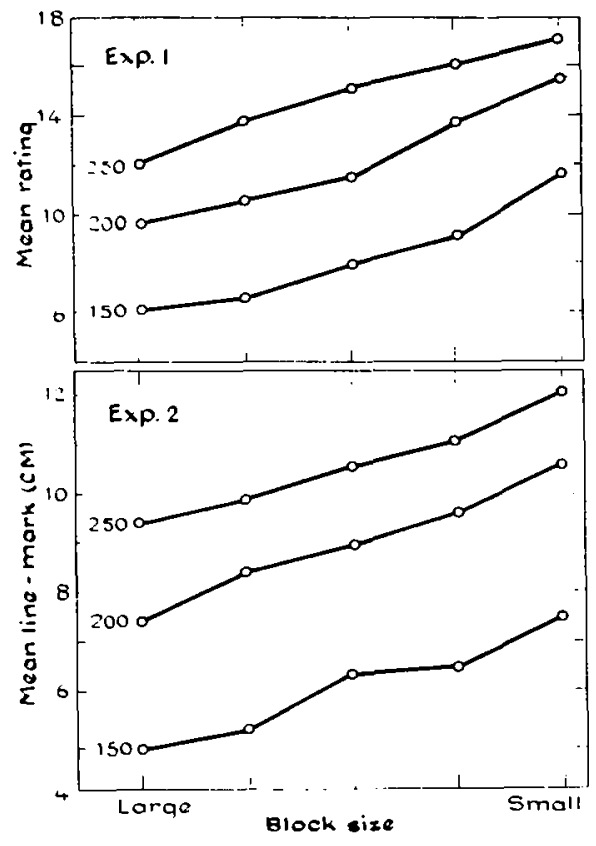

Fig. 1
Experiment 2. Perhaps the major reservation is that long-term data on single Ss would be required for a completely satisfactory test of the model.

\section{Density Interpretation}

\section{DISCUSSION}

Although the data provide support for the quantitative prediction, it is a more difficult question as to how far this supports the qualitative basis of the averaging formulation. It is appropriate, therefore, to point out that the data show a clear inconsistency with the hypothesis that Ss are really judging density. This is one of the more popular interpretations (see e.g., Helson, 1964; Huang, 1945; Ryan, 1940; Thouless, 1931). It is attractive for providing a physicalistic definition of the stimulus and not requiring an expectancy concept.

To test the density theory, it is desirable to put it into quantitative form. This may be done as follows:

$$
R=f_{0}(W)+f_{1}(W) / g(S)
$$

where $f_{0}$ and $f_{1}$ are functions of the physical weight, $W$, and $g$ is a function of the visual size, $S$. The functions $f_{0}, f_{1}$, and $g$ are assumed to be monotone but are otherwise unrestricted.

If $f_{1}(W)$ is taken proportional to the mass, $g(S)$ proportional to the volume, and $f_{0}(W)$ set equal to zero, then the right side of Eq. 4 is proportional to the density. Equation 4 thus includes the physical density formulation as a special case. Of course, neither $f_{1}(W)$ nor $g(S)$ need be required to have strict physical interpretations.

The primary qualitative consequence of Eq. 4 is a divergence prediction. If Eq. 4 is correct, then the curves of Fig. 1 should diverge to the right. This may be seen directly by noting that the derivative of $R$ with respect to $S$ is an increasing function of $W$. Perhaps of more interest is a geometrical proof that simultaneously demonstrates the applicability of a bilinear test (Anderson, 1970) to the density formulation. Consider the lowest curve, for $\mathrm{W}=150 \mathrm{~g}$, and imagine that the size values on the horizontal axis are moved laterally so that the five data points fall on a straight line. This can always be done, sampling error neglected. The first two points may be left as they are, and these two determine a straight line. Each remaining data point is then slid horizontally till it lies on this same straight line. In Fig. 1, the curves happen to be nearly linear so that only slight adjustments would be required.

The effect of this procedure is to produce a linear scale of $1 / g(S)$ on the horizontal axis. Equation 4 then implies that each remaining curve should also be a straight line, with slope $f_{1}(W)$, except for sampling error. The upper curves, for the heavier weights, should have greater slope. They should, therefore, diverge to the right. This derivation also holds if $f_{0}(W)$ is nonzero.

This divergence prediction is contrary to the data of Fig. 1. Experiment 1 shows a mild convergence to the right and Experiment 2 shows parallelism. The density theory is thus inconsistent with the data.

This same argument would also apply to certain of the expectancy theories. Any formulation that assumes that the lifted weight is judged, partly or wholly, as a proportion of, or relative to, the expected weight must also make the divergence prediction. This is discussed more fully below, under comparative illusions.

\section{Other Formulations}

Although the size-weight illusion has been under study for some time, there are not many data that bear directly on the present formulation. Scripture's (1897) equation expressing the magnitude of the illusion in terms of physical stimulus parameters is one of the few attempts at quantification, but its interest is mainly historical.

The report of Robinson (1964) has special interest, in part because of its very nice work on developmental trends in the illusion and in part for making a distinction parallel to that between the $w$ and $s^{*}$ parameters of Eq. 3. Robinson found that the magnitude of the illusion decreased with age, contrary to previous theory and data. He went on to show that younger children have poorer weight discrimination, and that training in weight discrimination decreased the illusory effect. In line with this, he then interpreted the developmental decrease to reflect a correspondingly greater reliance on the kinesthetic lift cues. This would correspond, in the present model, to a developmental decrease in the w parameter. Robinson also suggests that the size-weight expectancy, which would correspond to $s^{*}$, is fully developed at a very early age.

Two other articles, from somewhat different substantive areas, may also be relevant here. Jameson and Hurvich (1964) treat brightness and color contrast in terms of induced opponent processes. Their formulation leads to a subtractive model that has certain similarities to that used here. Whether there is any deeper substantive parallel to judgments of lifted weights, as in terms of opponent muscular processes, is hard to say. Price-Williams (1954; Experiment 2) found that time 
intervals were reproduced longer the farther apart were the two ights that signalled the beginning and end of the interval. His 3 by 5, Distarce by Duration design, yielded three parallel curve . ist as in the present Experiment 2 . This $m_{\omega}$, be a positive rather than a negative context effect. Both make the same pa:illelism prediction as noted below.

\section{Functional Measurement}

The present results also illustrate a general method of psychological scaling (Anderson, 1970). According to functional measurement theory, the row and column means of the factorial design constitute interval scales of the stimuli, weight and size. Thus, the near linearity of the curves of Fig. 1 indicates that $s *$ is approximately a linear function of the side length of the blocks; as plotted, the slope reflects the negative value of $w_{2}$ in Eq. 1. Similarly, the elevations of the three curves give the subjective heaviness values, $s$, up to a linear transformation. The subjective difference between 150 and $200 \mathrm{~g}$ appears somewhat greater than that between 200 and $250 \mathrm{~g}$. The present experiments were not designed to get a complete scale of subjective weight, for which considerably more weight values would be required. They do, however, illustrace the potential power of functional measurement for scaling quantities that would otherwise be elusive.

Negative, Positive, and Comparative Illusions

Although the present results are very tentative, they do indicate that integration theory provides a useful vantage point for the study of illusions. From this view, three classes of illusions are of special interest. This classification does not include all illusions, but it does include many stimulus complexes that are not ordinarily considered as illusions.

The first class, illustrated by the size-weight illusion studied here, may be called illusions of negative context. In these illusions, some secondary or contextual feature of the stimulus complex has a negative effect on the judgment. This contextual feature is integrated in to the judgment in the same manner as the primary property that is ostensibly being judged, only with a negative weight.

The second class, illusions of positive context, is defined analogously. Formally, these positive and negative illusions differ only in the sign attached to $w_{2}$ in Eq. 1, so the quantitative analysis is much the same for both. The Muller-Lyer illusion would seem to exemplify illusions of positive context, and the Sander parallelogram may fall in this class as well. From the present view, the judgment of the Müller-Lyer figure results from an averaging of the stimulus information, with the contextual wings receiving nonzero weight. This interpretation falls within the group of "total impression" theories of this illur: ". (Boring, 1942; Woodworth, 1938). It is conceptually similar to the judgmental interpretation given originally by Müller-Lyer himself $(1889,1896 a, b)$, that one's evaluation is involuntarily influenced by the space bounded by the wings. This could be given quantitative form by representing the judgment as a weighted average of the actual line and the imaginary parallels cut off by the wings. Auerbach and, especially, Brunot (cited in Titchener, 1901) have given rudimentary versions of an averaging model.

The terms "negative" and "positive context" have been used in preference to the customary terms, contrast and assimilation, for two reasons. In the first place, comparative illusions, discussed below, are also usually considered as contrast effects. But comparative illusions are quite different in nature from illusions of negative context. In the second place, the terms, contrast and assimilation, tend to carry the theoretical implication that the value of the primary stimulus property has been shifted from or toward the values of the contextual stimuli. This need not be true. Weighting the contextual features, here as elsewhere (Anderson, 1966), would produce the same result with no changes in stimulus value. The present terminology is neutral to the theoretical interpretation.

The third class includes illusions of comparative judgment as well as many cases that would not be considered as illusions. The distinguishing feature of this class is that the secondary stimulus property serves as a yardstick. The primary property is judged partly or wholly relative to this yardstick. Included here would be certain illusions of perspective, of relative size such as Ebbinghaus's figure (Luckiesh, 1922) as well as the Ponzo and Wundt illusions (e.8., Hochberg, 1964).

Although there is a not inconsiderable degree of agreement on the general nature of this class of illusions, only the workers in adaptation-level theory (e.g., Helson, 1964; Restle \& Merryman, 1968) have attempted much quantitative analysis. The paper of Hake, Faust, Mclntyre, and Murray (1967) is also of interest for its use of multiple discriminant analysis to handle choice data as opposed to the numerical response measures considered here.

The present analysis of comparative illusions is simple. At bottom, it rests on the idea (see, e.g., Gogel, 1968; Hake et al, 1967; Helson, 1964; Hochberg, 1964; Jastrow, 1892; Parducci, 1965; Rock \& Ebenholz, 1959) that the response results, at least in part, from the use of the referent stimulus as a direct yardstick of judgment.

'ith several referents, there would be several yardsticks, but only one will be considered here. The general model then becomes

$$
R=f_{0}(S)+f_{1}(S) / g_{1}(Y),
$$

where $f_{0}(S)$ and $f_{1}(S)$ are arbitrary functions of the physical stimulus property $S$ which is to be judged, and $g_{1}(Y)$ is an arbitrary function of the yardstick stimulus $Y$. The judgment is thus considered as the resultant of an absolute evaluation, $f_{0}(S)$, and a comparative evaluation, $\mathrm{f}_{\mathbf{1}}(\mathrm{S}) / \mathrm{B}_{1}(\mathrm{Y})$. It follows directly that larger values, $g_{1}(Y)$, of the referent stimulus produce smaller values of the response, a "contrast" effect.

This comparative judgment model leads to the same divergence prediction that was derived for Eq. 4 above. The proof is the same since Eqs. 4 and 5 have the same form. Furthermore, this divergence prediction can be given precise form in terms of a test of bilinearity (Anderson, 1970). Scaling of the stimuli is also possible with functional measurement procedures.

Three recent studies give rough quantitative support to this comparative judgment model. Ross and DiLollo (1969) obtained magnitude ratings of lifted weights relative to a standard presented once at the beginning of the experimental session. Ross and DiLollo give an extensive theoretical and experimental development, but only the preshift data of their Experiment 1 (Fig. 3) are immediately relevant here. The linearity of their curves implies directly the validity of the bilinear prediction of Eq. 5, at least within each series; whether the present formulation would account for the between-series differences is uncertain. In the report of Freeman and Adam (1965), the relation between the anchor and non-anchor curves would also seem to support the bilinear prediction.

Another interesting experimental illustration of comparative judgments is given by Helson and Kozaki (1968; Experiment 2). The judgments were ratings of sizes of squares, each preceded by an anchor stimulus of extreme size. The data of their Fig. 2 would follow the present integration model at least as well as the curves predicted from adaptation-level theory. A sharper comparison of the two formulations could probably be obtained by using more than one yardstick stimulus.

\section{REFERENCES}

ANDERSON, N. H. Application of an additive model to impression formation. Science, 1962 , 138, 817-818.

ANDERSON, $\mathrm{N}, \mathrm{H}$. Component ratings in 
impression formation. Psychonomic Science, $1966,6,279-280$.

ANDERSON, N. H. A simple model for information integration. In $R$. P. Abelson, $E$. Aronson, W. J. McGuire, T. M. Newcomb, M. J. Rosenberg, and P. H. Tannenbaum (Eds.), Theories of cognitive consistency: sourcebook. Chicago: Rand McNally, 1968.

ANDERSON, N. H. Functional measurement and psychophysical judgment. Psychological Review, 1970, in press.

BORING, E. G. Sensation and perception in the history of experimental psychology. New York: A ppleton-Century Crofts, 1942 .

FREEMAN, I., \& ADAM, J. Size-weight illusion and anchoring of weight judgments. Perceptual \& Motor Skills, 1956, 20, 63-68.

GOGEL, $W$. $C$. The measurement of perceived size and distance. In W. D. Neff (Ed.), Contributions to sensory physiology. Vol. 3. New York: Academic Press, 1968.

HAKE, H. W., FAUST, G. W., \& MCINTYRE, J.S., \& MURRAY, H. G. Relational perception and modes of perceiver operation. Perception \& Psychophysics, 1967, 2, 469-478.

HELSON, H. Adaptation-level theory. New York: Harper \& Row, 1964.

HELSON, H., \& KOZAKI, T. Effects of duration of series and anchor-stimuli on judgments of perceived size. American Joumal of Psychology, 1968, 81, 291-302.

HOCHBERG, J. E. Perception. Englewood Cliffs, N.J: Prentice-Hall, 1964.
HUANG, I. The size-weight illusion and the "weight-density illusion." The Journal of General Psychology, 1945, 33, 65-84.

JAMESON, D., \& HURVICH, L. M. Theory of brightmess and color contrast in human vision. Vision Research, 1964, 4, 135-154.

JASTROW, J. A study of Zöllner's figures and other related illusions. American Joumal of Psychology, 1892, 4, 381-398.

LUCKIESH, M. Visual illusions. New York: van Nostrand, 1922.

M ÜL LER-LYER, F, C. Optische Urtheilstauschungen. Archiv für Physiologie, $1889,263-270$ (plus appended figures).

MÜLLER-LYER, F. C. Zur Lehre von den optischen Tauschungen. Über Kontrast und Konfluxion. Zeitschrift fur Psychologie, $1896 a, 9,1-16$.

MÜLLER-LYER, F. C. Über Kontrast und Konfluxion (Zweiter Artikel). Zeitschrift für Psychologie, 1896b, 10, 421-431.

PARDUCCI, A. Category judgment: A range-frequency model. Psychological Review, $1965,72,407-418$.

PRICE-WILLIAMS, D. R. The kappa effect. Nature, 1954, 173, 363-364.

RESTLE, F., \& MERRYMAN, C. T. An adaptation-level theory account of a relative-size illusion. Psychonomic Science, 1968, 12, 229-230.

ROBINSON, H. B. An experimental examination of the size-weight illusion in young children. Child Development, 1964, 35, 91-107.

ROCK, I., \& EBENHOLTZ, S. The relational determination of perceived size. Psychological Review, 1959, 66, 387-401.

ROSS, J., \& DilOLLO, V. A vector model for psychophysical judgment. Journal of Experimental Psychology, 1968, 77, Monograph Supplement No. 3.

RYAN, T. A. Interrelations of the sensory systems in perception. Psychological Bulletin, $1940,37,659-698$

SCRIPTURE, E. W. The new prychology. New York: Scribner's, 1897.

THOULESS, R. H. Phenomenal regression to the 'real' object. II. British Journal of Psychology, 1931, 22, 1-30.

TITCHENER, E. B. Experimental psychology: $A$ manual of laboratory practice. Vol. 1, Part 2. Instructor's manual. New York: Macmillan, 1901.

WOODWORTH, R. S. Experimental psychology. New York: Henry Hoit, 1938.

\section{NOTES}

1. Address: Department of Psychology, University of California, San Diego, La Jolla, California 92037.

2. I wish to thank Kurt Richards for his assistance with these experiments. This work was supported by NSF Grant GB-6666 and by the Center for Advanced Study in the Behavioral Sciences.

(Accepted for publication Ocrober 1, 1969.) 